\title{
Dementia in the COVID-19 Period
}

\author{
Amos D. Korczyn \\ Department of Neurology, Tel Aviv University, Tel Aviv, Israel
}

Accepted 26 May 2020

While we are witnessing the huge effects of the present corona pandemic, let us not forget that large as it is, its size is small relative to that of dementia, labeled by the World Health Organization as a public health priority. Yet, the huge investments in the COVID-19 illness are much higher than those dedicated to dementia research and treatment. This is in spite of the fact that the SARS-CoV-2 will disappear soon, like previous Corona viruses, or an immunization against it will be developed. On the other hand, the dementia epidemic is getting bigger all the time because of the aging of the population, prolonged survival, and other factors.

However, the effects of the COVID-19 infection on those older people who have cognitive decline (due to Alzheimer's disease or other causes) need to be addressed. This is not restricted to those cognitively impaired people who happen to become infected by the virus. The draconic measures inflicted on the population at large impose an disproportionate burden on the elderly and particularly on those with cognitive impairment. The restrictions to move about and meet friends and family members are incomprehensible to them. The explanations given are not well understood and, in any case, soon forgotten, leading to stress on patients and caregivers.

The risk factors for dementia in general, age, obesity, cardiovascular disease, hypertension, and diabetes mellitus, are also the major risk factors for COVID-19, making persons with cognitive impair-

\footnotetext{
${ }^{*}$ Correspondence to: Professor Amos D. Korczyn, MD, MSc, Tel Aviv University, Ramat Aviv 69978, Israel. E-mail: E-mail: amoskor@tauex.tau.ac.il.
}

ment particularly vulnerable to the SARS-CoV-2 infection, and quite likely making the disease more severe. Therefore, these people need to maintain rigid control, and this applies even more to those who reside in retirement facilities or nursing homes. The availability of virtual communication such as Skype or Zoom facilitates contact with those persons [1], although we should not forget that physical contact is very important and can only partially be replaced by electronic communication, even more so since the elderly are computer-naïve and because of cognitive limitations may have difficulties in absorbing and being able to incorporate new technologies. In any case, the imposed physical isolation should not imply also social isolation. In fact, human contact is so important that it should be encouraged and enhanced.

The effects of the imposed isolation are likely to induce or exacerbate depression, anxiety, and stress, possibly reflected by aggression. The stress is considered to be detrimental to people with cognitive impairment [2] and may lead to a cytokine release syndrome which is potentially dangerous, and needs to be avoided. It is obvious that alleviating measures should be implemented, as far as possible, before the stress is expressed. Most relevant are behavioral measures.

Once patients with cognitive impairment, mild, or more severe dementia are diagnosed with COVID19 , a critical question is whether they need to be hospitalized. Whatever their condition, there is a chance that they will be transferred to an intensive care facility where they will be isolated and treated by unfamiliar persons wearing masks and dressed in astronauts-like cloths. There is little time 
for explanations and this is particularly true for those people whose understanding is impaired. Many of them will develop restlessness, agitation, and attempt to free themselves, requiring pharmacological sedation, which will further impair their oxygenation leading to intubation under anesthesia. The consequences of prolonged anesthesia are dire. Mortality is high and the survivors may show cognitive deterioration and possibly post-traumatic stress disorder.

Unfortunately, only a minority of people with cognitive impairment have signed advanced directive forms. Even if they have mild cognitive impairment, by the time they arrive at the hospital door it may be too late to discuss the issue with them. The time available is too short, and they are under stress as well as possibly hypoxemic which is not conductive to have a serious discussion aiming to receive an informed consent to any intervention. Given the harsh prognosis once admitted to hospital, it may be better if people with cognitive impairment who develop COVID-19 remain at their residence and be treated there $[3,4]$.

\section{DISCLOSURE STATEMENT}

The author's disclosure is available online (https:// www.j-alz.com/manuscript-disclosures/20-0609).

\section{REFERENCES}

[1] Bloem BR, Dorsey ER, Okun MS (2020) The coronavirus disease 2019 crisis as catalyst for telemedicine for chronic neurological disorders. JAMA Neurol, doi: 10.1001/jamaneurol.2020.1452.

[2] Simard M, Hudon C, van Reekum R (2009) Psychological distress and risk for dementia. Curr Psychiatry Rep 11, 41-47.

[3] Zagury-Orly I, Schwartzstein RM (2020) Covid19 - a reminder to reason. $N$ Engl $J$ Med, doi: 10.1056/NEJMp2009405.

[4] Arya A, Buchman S, Gagnon B, Downar J (2020) Pandemic palliative care: Beyond ventilators and saving lives. CMAJ 192, E400-E404. 